Notes : (1) On a Geometrical Problem.

(2) On an Algebraical Equation of Professor Cayley's.

By Professor StegancL.

\title{
On the Fundamental Principles of Quaternions and other Vector Analyses.
}

By Dr William Peddie.

When a student of mathematics commences the study of a subject which involves the assimilation of what are, to him, fundamentally new ideas, his progress is, as a rule, slow at first. And, even after he has become accustomed to these ideas, he may still require a Iong course of laborious practice, before he can attain to that mastery of the method which would enable him to use it as a powerful aid to research. Thus students, familiar with geometrical methods, when first commencing the study of Cartesian analysis, require much practice before they can call up mentally the geometrical figure corresponding to a given equation. And, the more general the new method is, the greater is the difficulty felt to be. So, in Hamilton's system of quaternions, the difficulty of assimilation is greater than it is in the Cartesian analysis. And it seems as if it were for this reason that, in recent years, attempts have been made, by men of known mathematical ability, to smooth the paths.

Practically, all these attempts consist in using, instead of Hamilton's, another system of quaternions, cut up into parts; the parts of that system being used because they are imagined to be superior to the corresponding parts of Hamilton's system in respect of naturalness. Subsequently, I shall say somewhat regarding the reasonableness (or unreasonableness) of this clain; but, whatever conclusion be accepted on this point, M'Auley's appeal to the spoon-feeders, to "provide spoon-meat of the same kind as the other physicians" (Nature, Dec. 15, 1892), is most appropriate.

Some of the strictures recently passed on quaternions refer rather to the way in which the subject is presented in the standard treatises than to quatemions themselves. Heaviside (Electrician, Nov. 18, 1892) refers to three special "sticking-points" in Tait's treatise. One of these is the investigation of Hamilton's cubic 